\title{
A Calorie-Restricted Diet Decreases Brain Iron Accumulation and Preserves Motor Performance in Old Rhesus Monkeys
}

\author{
Erik K. Kastman, ${ }^{1,2 *}$ Auriel A. Willette, ${ }^{2,3,4 *}$ Christopher L. Coe, ${ }^{3,4}$ Barbara B. Bendlin, ${ }^{1,2}$ Kris J. Kosmatka, ${ }^{1,2}$ \\ Donald G. McLaren, ${ }^{1,2,5}$ Guofan Xu, ${ }^{1,2}$ Elisa Canu, ${ }^{1,2}$ Aaron S. Field, ${ }^{6}$ Andrew L. Alexander, ${ }^{4}$ Mary Lou Voytko, ${ }^{7}$ \\ T. Mark Beasley, ${ }^{8}$ Ricki J. Colman, ${ }^{8}$ Richard H. Weindruch, ${ }^{1,2,9}$ and Sterling C. Johnson ${ }^{1,2,4,9}$ \\ ${ }^{1}$ Geriatric Research Education and Clinical Center, Wm. S. Middleton Memorial Veterans Hospital, Madison, Wisconsin 53705, ${ }^{2}$ Wisconsin Alzheimer's \\ Disease Research Center, University of Wisconsin School of Medicine and Public Health, Madison, Wisconsin, 53705, ${ }^{3}$ Harlow Primate Laboratory, \\ Department of Psychology, University of Wisconsin, Madison, Wisconsin 53715, ${ }^{4}$ Waisman Imaging Center, University of Wisconsin, Madison, Wisconsin, \\ 53705, ${ }^{5}$ Neuroscience Training Program, University of Wisconsin, Madison, Wisconsin 53706, ${ }^{6}$ Department of Radiology, University of Wisconsin, \\ Madison, Wisconsin, 53792, 7 Department of Neurobiology and Anatomy and Interdisciplinary Neuroscience Program, Wake Forest University School of \\ Medicine, Winston-Salem, North Carolina 27157, ${ }^{8}$ Department of Biostatistics, University of Alabama at Birmingham, Birmingham, Alabama 35294, and \\ ${ }^{9}$ Wisconsin National Primate Research Center, Madison, Wisconsin 53792
}

Caloric restriction (CR) reduces the pathological effects of aging and extends the lifespan in many species, including nonhuman primates, although the effect on the brain is less well characterized. We used two common indicators of aging, motor performance speed and brain iron deposition measured in vivo using magnetic resonance imaging, to determine the potential effect of CR on elderly rhesus macaques eating restricted ( $n=24,13$ males, 11 females) and standard ( $n=17,8$ males, 9 females) diets. Both the CR and control monkeys showed age-related increases in iron concentrations in globus pallidus (GP) and substantia nigra (SN), although the CR group had significantly less iron deposition in the GP, SN, red nucleus, and temporal cortex. A Diet $\times$ Age interaction revealed that $\mathrm{CR}$ modified age-related brain changes, evidenced as attenuation in the rate of iron accumulation in basal ganglia and parietal, temporal, and perirhinal cortex. Additionally, control monkeys had significantly slower fine motor performance on the Movement Assessment Panel, which was negatively correlated with iron accumulation in left $\mathrm{SN}$ and parietal lobe, although $\mathrm{CR}$ animals did not show this relationship. Our observations suggest that the CR-induced benefit of reduced iron deposition and preserved motor function may indicate neural protection similar to effects described previously in aging rodent and primate species.

\section{Introduction}

Caloric restriction (CR), a reduction of calories without changes in essential nutrient intake, reduces the pathological effects of aging and extends the lifespan in many species, including yeast and nematodes (Weindruch and Walford, 1988), rodents (Masoro, 2005) and nonhuman primates (Mattison et al., 2007; Colman et al., 2009). CR diets started in middle-aged rhesus monkeys reduce overall mortality and morbidity, sarcopenia

\footnotetext{
Received May 28, 2012; accepted May 28, 2012.

This work was supported by funding from the NIH to the Wisconsin National Primate Research Center (WNPRC) (Grants P01 AG11915 and P51 RR000167) and National Institute on Aging Grant AG000213. This research was conducted in part at a facility constructed with support from Research Facilities Improvement Program Grants RR15459-01 and RR020141-01. The project also benefited from resources and facilities at the Wm. S. Middleton Memorial leterans Hospital. We gratefully acknowledge the excellent technical assistance provided by R. Fisher, $S$. Baum, J. Smith, J. Adriansjach, C. Armstrong, the Waisman Center, and the Animal Care, Veterinary and Pathology Staff of the WNPRC, EVP, and thank Dr. Craig Atwood, Ph.D. for critical comments on the manuscript.

*E.K.K. and A.A.W. contributed equally to the manuscript.

Correspondence should be addressed to Sterling C. Johnson, Geriatric Research Education and Clinical Center, D-4225 Veterans Administration Hospital, 2500 Overlook Terrace, Madison, WI 53705. E-mail: scj@medicine.wisc.edu.

DOI:10.1523/JNEUROSCI.2553-12.2012

Copyright $\odot 2012$ the authors $\quad 0270-6474 / 12 / 3211897-08 \$ 15.00 / 0$
}

(Colman et al., 2008), and insulin resistance (Gresl et al., 2001) possibly via decreases in oxidative stress (Wanagat et al., 1999; Forster et al., 2000) or alterations in internal metabolic and signaling pathways (Masoro et al., 1992; Anson et al., 2003; Masoro, 2005; Qin et al., 2006). Although less understood, the effects of $\mathrm{CR}$ on the brain are similarly protective: CR increases dendritic spine density (Stranahan et al., 2009), increases astrocytic functions including glutamate uptake (Ribeiro et al., 2009), reduces cortical amyloid deposition (Mouton et al., 2009), and attenuates iron accumulation (Xu et al., 2008) after CR diet in both young and old rodents.

One cross-species marker of aging in the brain is an increased concentration of non-heme iron (Dhenain et al., 1997; Zecca et al., 2001, 2004a; Péran et al., 2007). The concentration and distribution of iron accumulation have been well established histologically, with the highest rates of deposition near striatal regions, globus pallidus (GP), and substantia nigra (SN), and moderate accumulation in cortical gray matter with relative sparing of white matter (Hallgren and Sourander, 1958; Koeppen, 2003; Haacke et al., 2005). Much of this non-heme brain iron is stored in ferritin, a spherical protein that sequesters crystallized iron in 
Table 1. Animal characteristics

\begin{tabular}{lll}
\hline & Control & Diet restricted \\
\hline$N$ & 17 & 24 \\
Sex (male/female) & $9 / 8$ & $13 / 11$ \\
Age (years) & $23.3 \pm 0.7$ & $24.6 \pm 0.5$ \\
Total Brain Volume (ml) & $85.72 \pm 1.9$ & $81.83 \pm 2.6$ \\
\hline
\end{tabular}

Age and Total Brain Volume per group are represented by the mean and SEM.

high local concentrations (Martin et al., 1998; Zecca et al., 2004a). Iron stored in ferritin causes magnetic field inhomogeneities, shortening the transverse relaxation time constant $\mathrm{T} 2$ and increasing the relaxation rate (R2) of nearby protons, creating a signature signal hypointensity on T2-weighted MR images (House et al., 2007). Several histological investigations have demonstrated high correlations between iron concentration and iron deposition measured with MR (Drayer et al., 1986; Bizzi et al., 1990; Bartzokis et al., 1997; Gelman et al., 1999; Hardy et al., 2005), resulting in an acceptance of T2 or its inverse, R2, as a biomarker of brain aging (Schenck and Zimmerman, 2004). A second common measure of the progression of aging is motor performance, which decreases with age in humans and monkeys (Gash et al., 1999). Perhaps not coincidentally, iron is higher in brain regions associated with motor function such as GP and SN.

The primary aim of these analyses was to determine the potential effect of CR on brain iron and motor function in aged rhesus monkeys. We hypothesized that CR would attenuate the normal effects of age on brain iron deposition, as indexed by decreased $\mathrm{T} 2$ or increased $\mathrm{R} 2$, in iron-rich regions including the nigrostriatal pathway. We also hypothesized that CR animals would exhibit better manual dexterity and faster fine motor performance and that motor speed would be related to iron load in subcortical areas, such as GP.

\section{Materials and Methods}

Animals. Forty-one rhesus monkeys (Macaca mulatta) between the ages of 18 and 31 years were scanned and included in the analyses (Table 1). The animals were all from the Dietary Restriction and Aging Study at the Wisconsin National Primate Research Center (WNPRC). Length of the CR diet ranged from 12 to 17 years, and was initiated when animals were in middle age. Details of the protocol have been reported previously (Kemnitz et al., 1993). CR animals ( $n=17 ; 8$ males, 9 females) were fed $30 \%$ less chow than control animals ( $n=24$; 13 males, 11 females) of a similarly formulated diet supplemented to achieve comparable vitamin and mineral content between $\mathrm{CR}$ and controls. Ferric citrate within the mineral mix was the only source of iron in the diet $(0.12 \%$ of total chow composition). The CR and control diets respectively contained $294 \mathrm{mg} / \mathrm{kg}$ and 206 $\mathrm{mg} / \mathrm{kg}$ iron. The rooms were maintained on a $12 \mathrm{~h}$ light/dark cycle, and scans were typically conducted during the morning hours. The magnetic resonance imaging (MRI) scans of 7 additional monkeys were excluded from analysis due to poor quality images caused by head movement despite the anesthesia. The research protocol was approved by the Institutional Animal Care and Use Committee, and the WNPRC is fully accredited by the Association for Assessment and Accreditation of Laboratory Animal Care.

Dietary iron intake and peripheral iron. Food intake for each animal was calculated daily, and the average amount of iron consumed from a chow ration per week was calculated for each animal over a 2 year period around the time of scan to achieve a complete view of iron intake during the study. To characterize iron uptake and assess whether circulating iron is related to ferritin-bound brain iron, serum levels of iron were assayed. Whole blood was centrifuged to derive serum and a standard chemistry panel was then run on a COBAS INTEGRA analyzer (Meriter Laboratories). Relevant hematology measures such as hematocrit (Hct), hemoglobin ( $\mathrm{Hgb})$, and mean corpuscular volume (MCV) were examined near the time of scan to determine whether monkeys exhibited characteristics of anemia or iron overload (Kemnitz et al., 1993).

MRI acquisition. The monkeys were scanned on a GE 3T Signa scanner using a quadrature transmit-receive volume coil with $18 \mathrm{~cm}$ diameter. The axial T2 relaxation scan was a multiple-slice, four-echo, spin-echo pulse sequence with four echoes at echo time $(\mathrm{TE})=15,30,45$, and 60 ms. Other parameters included: $\mathrm{TR}=2000 \mathrm{~ms}$, flip angle $(\mathrm{FA})=90^{\circ}$, field of view $(\mathrm{FOV})=160 \mathrm{~mm}$, matrix $=256 \times 256$, slice thickness $=1.7$ $\mathrm{mm}$. This sequence resulted in a voxel size of $0.625 \times 0.625 \times 1.7 \mathrm{~mm}$ across 24 slices. The slice prescription was such that there was full coverage of deep gray structures and temporal lobes. A whole-brain T2weighted Fast Spin Echo (FSE) $\left(\mathrm{TE}=88.5 \mathrm{~ms}, \mathrm{TR}=4500 \mathrm{~ms}, \mathrm{FA}=90^{\circ}\right.$, $\mathrm{FOV}=160 \mathrm{~mm}$, matrix $=512 \times 512$, slice thickness $=1.7 \mathrm{~mm})$ or a T2-weighted 3D FSE $\left(\mathrm{TE}=81.556, \mathrm{TR}=2300 \mathrm{~ms}, \mathrm{FA}=55^{\circ}, \mathrm{FOV}=140\right.$ $\mathrm{mm}$, matrix $=512 \times 512)$ was also acquired and used during processing to achieve accurate normalization to a T2-weighted atlas (McLaren et al., 2009). Additionally, a whole-brain T1-weighted scan (parameters reported by McLaren et al., 2010) was acquired to aid in skull-stripping the brains. Animals were anesthetized before the scan with ketamine (up to $15 \mathrm{mg} / \mathrm{kg}$ ) and xylazine (up to $0.6 \mathrm{mg} / \mathrm{kg}$ ).

Absolute and accurate T2 measurements with multiple-echo pulse sequences can be optimized using slice-selective refocusing pulses and crushers for removing stimulated echoes (see Poon and Henkelman, 1992 for a review of T2 mapping issues) and stimulated echoes can cause the T2 to be overestimated. However, the signal obtained in this study is modulated primarily by $\mathrm{T} 2$ and, based on previous studies, is known to correlate highly with absolute T2 measurements.

Image analysis. The transverse relaxation rate, $\mathrm{T} 2$, was calculated by first performing log transformations of the voxel signal intensity data for all 4 TEs and then linear-fitting the slope according to the monoexponential Spin Echo signal intensity equation (Poon and Henkelman, 1992).

SPM5 was used for all coregistration and normalization steps (Ashburner and Friston, 2000; Mechelli et al., 2005; Ridgway et al., 2008). T2 Relaxation maps for each animal were normalized to our T2weighted Rhesus Macaque atlas (McLaren et al., 2009) to obtain a common space for voxelwise analysis using a three-stage process. First, we estimated the transformation matrix needed to coregister the TE45 volume of the echo relaxation image to the T2-weighted image from the same individual using a 6-parameter transformation. Next, we estimated the transformation matrix needed to spatially normalize that individual's T2-weighted image to a previously published T2weighted atlas using a 12-parameter affine transformation. The T2weighted atlas template (McLaren et al., 2009) was created from nine monkeys (none of which overlapped with the current analysis) and is registered with the histological atlas of Saleem and Logothetis (2007). No nonlinear transforms were performed because the reduced variability of the rhesus brains fit the atlas space within approximately a millimeter without them. Finally, the two transformation matrices were combined (i.e., TE45 $\rightarrow$ T2 and T2 $\rightarrow$ T2 atlas) to warp the T2 relaxation maps to $112 \mathrm{RM}$-SL atlas space and were re-sliced to 0.5 $\mathrm{mm}$ isotropic voxels. T2-weighted images were manually de-skulled using a brain mask traced from high-resolution T1 images acquired during the same scan session before normalization to reduce the impact of skull information on the registration between brains.

Full factorial analyses of covariance (ANCOVAs) of the T2 images were conducted in SPM5 to examine the main effects of Age and Diet Group, as well as the Age $\times$ Diet Group interaction (Ashburner and Friston, 2000). Sex and Total Brain Volume (TBV) were included as covariates. The effect of sex was controlled for because some studies suggest females have less brain iron accumulation (Bartzokis et al., 2007). To minimize false-positive results, an omnibus $F$-contrast for Age, Diet Group, and Age $\times$ Diet Group at a liberal voxelwise threshold of $\alpha=0.05$ (uncorrected) was used to mask all further contrasts. Separate one-tailed $t$ tests were then conducted to examine: (1) the negative association of Age, based on the prediction that T2 should decrease with age (i.e., increased iron accumulation); (2) whether the 
CR group had increased T2, based on the prediction that CR should attenuate iron accumulation; and (3) the Age $\times$ Diet Group interaction, where control animals would show the expected decrease in T2 signal per year of age versus no such relationship for CR monkeys. TBV was calculated from automated segmentation of the T2weighted images using FAST in FSL (Zhang et al., 2001) and entered in the model to account for any possible confounding influence of global volume on relaxation signal.

A voxel threshold of $\alpha=0.005$ (uncorrected) was used for all contrasts based on a priori regional hypotheses involving specific subcortical structures and T2. Multiple-comparison correction was performed using estimates from a Monte Carlo simulation with AlphaSim (http://afni.nimh.nih.gov/pub/dist/doc/manual/AlphaSim. pdf) to achieve a corrected cluster-level $\alpha=0.05$ (Forman et al., 1995). The Monte Carlo simulation estimates the probability of generating a cluster with 680 voxels based on randomly computed images with the same dimensions, voxel probability threshold, and smoothness parameters as MRI images input for analysis, which can be used as a threshold to remove small clusters likely to be significant only by chance. Although violation of non-stationarity is a concern for cluster correction of imaging data, our use of a $4 \mathrm{~mm}$ FWHM kernel does not appreciably bias the true $\alpha$ when using this permutational estimate of cluster size (Hayasaka et al., 2004).

Motor performance testing. We used an automated monkey Movement Assessment Panel (mMAP) testing system (Gash et al., 1999) to test the motor performance times of the animals retrieving small food items from a receptacle chamber. A day's testing session consisted of 12 trials, with equal trials between the right and left hand. The trial was initiated when the tester placed a small food item (e.g., a piece of cereal) into the center of the food receptacle. The trial was completed when the monkey removed the food from the receptacle and withdrew its hand back into the home cage. There was a $30 \mathrm{~s}$ delay before the next trial was initiated. The process was repeated until six trials had been completed with each hand. Monkeys were given $45 \mathrm{~s}$ to retrieve food before the trial was aborted.

Three tests of increasing level of difficulty were evaluated for each animal. The platform task (level I difficulty) required the removal of a small food item from a level platform in the food receptacle. The straight rod task (level II) involved maneuvering the food item up a straight rod. The q-mark task (level III) required the animal to thread the food item over a question mark-shaped rod in the receptacle. Each task was tested for $10 \mathrm{~d}$ before progressing to the next level of difficulty. Mean manual performance speed was computed for each session for: (1) the amount of time it took a subject to initially move its hand into the outer testing chamber ("reaction time," or RT); (2) the time spent in the outer chamber ("coarse" movement); and (3) the time spent removing the object from the inner chamber and withdrawing the hand ("fine" movement). Motor performance of a given animal, for each task, was divided into an "acquisition" phase during which the animals learned the task and improved their performance with each trial and a subsequent "proficiency" phase during which the animals' performance reached an asymptote. Task proficiency was determined by examining when a subject-specific asymptote in performance occurred throughout the consecutive series of 12 trials per session; a scree test was used for this determination (Cattell, 1966).

Behavioral and peripheral iron statistics. Multivariate repeatedmeasures analyses of covariance (MANCOVAs) were used as omnibus analyses to test RT, coarse, and fine motor performance for each of the tasks (platform, rod, q-mark). ANCOVA was used for post hoc testing between controls and CR monkeys. ANOVA tested differences in dietary iron intake, peripheral serum iron, and hematology variables. To explore whether CR-induced changes in central iron and motor performance were related, one-tailed split-level correlations were conducted between motor performance indices and regional T2 signal changes that were statistically different between CR and control monkeys. Maximal values of clusters generated from the Diet Group or Age $\times$ Diet Group analyses were used as regressors to test for associations between regional iron deposition and behavior. We predicted that controls would show slower motor performance as central iron increased in these maximal cluster values, whereas CR animals would not show this relationship. All behav-
Table 2. Age correlation cluster statistics

\begin{tabular}{lllr}
\hline Region & Voxel T & Cluster size & $x, y, z(\mathrm{~mm})$ \\
\hline Left globus pallidus & 4.05 & 783 & $-10,20,14$ \\
Right globus pallidus & 3.43 & 683 & $8,21,14$ \\
\hline
\end{tabular}

ioral analyses included Age and Sex as covariates. Alpha was set at 0.05. Analyses were conducted using SPSS 16.0.

\section{Results}

There was no significant Age, TBV, or difference in percentages of males and females between the two dietary groups (Table 1). There were no differences in hematology characteristics (Hgb, Hct, MCV) between the two groups as assessed with the blood panel after an initial year of CR (Kemnitz et al., 1993) and proximal to the time of scan.

\section{Effect of diet on dietary iron intake and peripheral serum iron} Based on mineral composition of the chow and the average amount of chow eaten per week, CR monkeys consumed significantly more iron per week than control monkeys (CR, $34.37 \pm 1.31 \mathrm{mg} /$ week; Control, $29.52 \pm 1.48 \mathrm{mg} /$ week) $\left(F_{(1,39)}=5.45, p<0.05\right)$. However, monkeys on CR $(152.4 \pm$ $5.81 \mu \mathrm{g} / \mathrm{dl})$ and control $(167.7 \pm 10.4 \mu \mathrm{g} / \mathrm{dl})$ diets had similar levels of circulating serum transferrin-bound iron $\left(F_{(1,39)}=\right.$ $1.48, p=0.231)$. This result suggests that despite greater iron consumption, there were no overt differences in iron uptake from the diet and peripheral partitioning of iron between tissue and the blood compartment.

Across all subjects, dietary iron intake was not significantly associated with peripheral serum iron or central iron accumulation that differed as a function of dietary condition (see below). Yet, higher levels of circulating serum iron were associated with higher T2 signal in SN within the left $[\mathrm{r}(41)=0.34, p<0.05]$ and right $[\mathrm{r}(41)=.36, p<0.05]$ hemispheres. The relationship was not significant with other regions of interest (ROIs) that differed due to dietary condition (see below).

\section{T2 correlations with Age}

In a combined analysis of CR and control monkeys, we found a significant negative correlation between Age and T2, indicating an increase in iron deposition in older animals, in several deep gray matter structures including the GP and SN bilaterally (Table 2, Fig. 1). The opposite contrast, where Age was positively correlated with T2, revealed clusters predominantly within CSF spaces adjacent to the midbrain and the parietal lobes. One possible explanation for this finding is that CSF has a shorter relaxation time than brain tissue and that the results suggest age-related atrophy in these areas.

\section{T2 differences between Diet Groups}

We next investigated the effect of the two dietary conditions on T2. Brain regions in which CR monkeys had higher T2 values than controls (i.e., less iron accumulation) included the GP, upper brainstem near red nucleus (RN), SN, and temporal cortex (Fig. 2). The significance and extent of each cluster can be found in Table 3, where the coordinates are reported in 112RM-SL space. There were no significant regions in which controls had higher T2 than CR monkeys.

\section{T2 Diet Group by Age interactions}

Finally, we investigated the direct effect of diet on the age gradient decline in $\mathrm{T} 2$ to examine the protective effect of CR, 
which would emerge as a significant interaction between Age and Diet Group. Regions in which Age and T2 correlations had a significantly steeper slope for controls compared with CR monkeys were the parietal and temporal cortices, striatal regions, $\mathrm{SN}, \mathrm{RN}$, and hippocampus (Fig. 3, Table 4).

\section{mMAP motor performance}

The motor performance of all 41 monkeys was evaluated. However, a subset of animals did not finish the full set of tasks: 33 animals completed the platform and rod tasks, and 28 completed the q-mark task. Those subjects unable or unwilling to perform did not differ by dietary group (Controls, 7; CR, 6). All animals that completed tasks were included in the analyses.

A repeated-measures MANCOVA first examined all motor indices for a given task (platform, rod, q-mark) during both initial acquisition and asymptotic proficiency. A Motor Task by Dietary Group interaction indicated that $\mathrm{CR}$ monkeys performed the q-mark tasks more quickly overall than controls $\left(F_{(5,125)}=4.26, p<\right.$ $0.05)$, but not the platform or rod tasks (data not shown). A subsequent omnibus tested whether or not groups exhibited differing motor performance for any of the motor indices (RT, coarse, fine) during acquisition and proficiency phases across the three mMAP tasks. For fine motor movement, a Motor Index by Dietary Group interaction requiring Huynh-Feldt correction indicated that CR monkeys were faster overall for the motor components of each task $\left(F_{(2.02,125)}=3.68, p<0.05\right)$. Post hoc analyses across motor indices and tasks were then conducted. Subjects had similar RT, coarse, and fine motor times for the platform and rod tasks (Fig. 4). To account for the influence of sustained motivation and attention during the q-mark task for RT, coarse, or fine motor movement, acquisition and proficiency performance during the platform and rod tasks were included as covariates. RT and coarse movement did not differ between groups in the q-mark task. CR monkeys performed fine motor movements in the inner chamber faster during the acquisition $\left(F_{(1,22)}=4.72, p<0.05\right)$ and proficiency $\left(F_{(1,22)}=5.42, p<0.05\right)$ phases of the task.

\section{T2 and q-mark motor performance associations}

Given that only fine motor performance for the q-mark task differed between groups (Fig. 5), exploratory split-level correlations were conducted to assess the extent to which lower T2 among Dietary Group or Age $\times$ Dietary Group ROIs were associated with this change. For ROIs related to the main effect of Dietary Group, controls with lower T2 in the left SN took longer to perform the task during the acquisition phase $[r(10)=-0.59, p<0.05] ;$ CR monkeys did not show this

\section{Effect of Age}
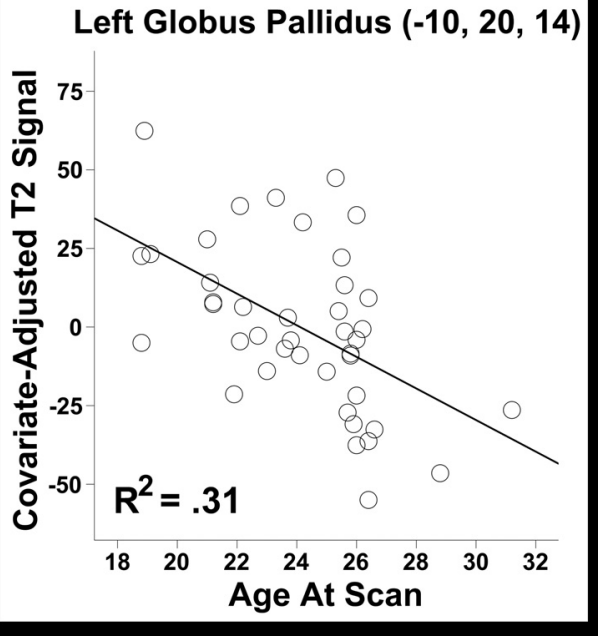

Figure 1. The correlation of Age at scan and T2 signal. Statistical parametric map of significant decreases in T2 signal were found within bilateral globus pallidus. The maximum voxel in the left hemisphere is plotted and depicts the main effect. As Age increases, T 2 signal decreases and represents increased iron concentration. The voxel and cluster thresholds were, respectively, $p<0.005$

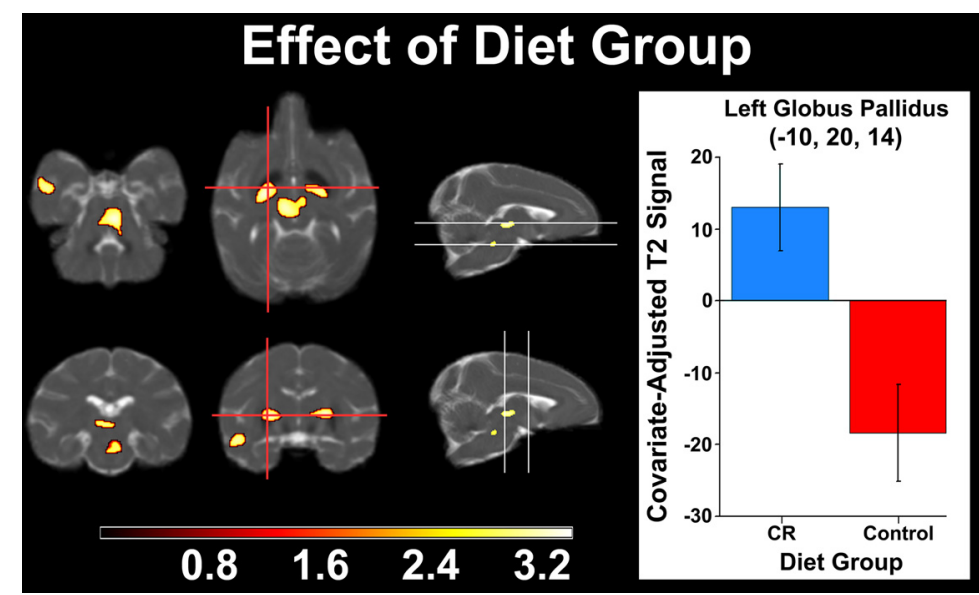

Figure 2. T2 relaxation signal differing by Diet Group. Statistical parametric map indicating decreased T2 signal (i.e., increased iron deposition) in control versus (R monkeys in basal ganglia, red nucleus, substantia nigra, and temporal cortex. A peak voxel in left globus pallidus represents the main effect of Group as bar graphs. Lower T2 values among controls correspond to increased brain iron. The voxel and cluster thresholds were, respectively, $p<0.005$ (uncorrected) and $p<0.05$ (corrected).

relationship $[r(18)=0.30, p=0.11]$. A similar pattern of associations was seen in an Age $\times$ Dietary Group cluster encompassing right dorsal superior temporal sulcus, where controls with lower T2 values also had slower fine motor acquisition performance $[r(10)=-0.56, p<0.05]$. This effect was not seen in CR monkeys $[r(18)=-0.20, p=0.31]$.

\section{Discussion}

Several measures of aging in old monkeys, including sarcopenia, poor glucoregulation, and atrophy of gray matter in the CNS are favorably affected by a CR diet (Gresl et al., 2003; Colman et al., 2008, 2009; Anderson et al., 2009). Less is known, however, about CR's protective effect on two other markers of brain aging: the accumulation of iron and deteriorating motor performance. Iron concentrations increase naturally with age in deep nuclei (striatum, GP, SN, and RN) in both humans (Bartzokis et al., 2007; 
Table 3. Dietary Group difference cluster statistics

\begin{tabular}{llcc}
\hline Region & Voxel T & Cluster Size & $x, y, z(\mathrm{~mm})$ \\
\hline Red nucleus & 3.43 & 1170 & $2,4,4$ \\
Left globus pallidus & 3.3 & 765 & $-11,14,13$ \\
Right globus pallidus & 3.28 & 703 & $10,16,14$ \\
Left substantia nigra & 3.21 & 788 & $-4,10,13$ \\
\hline
\end{tabular}

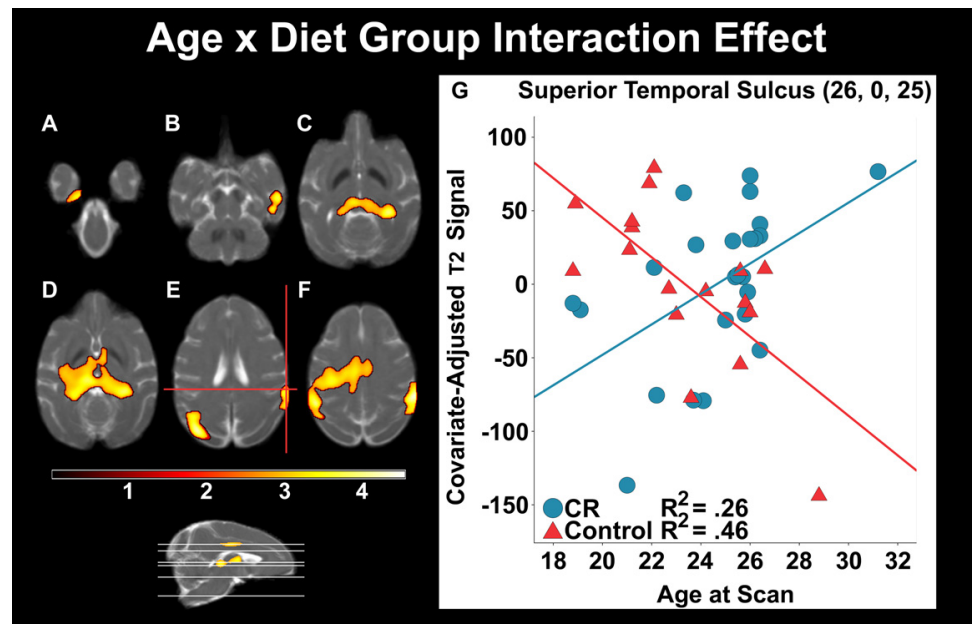

Figure 3. The differing association of T2 relaxation signal and Age as a function of Dietary Group. As Age increased per year, controls showed significantly lower T2 signal (i.e., increased iron deposition) versus Diet Group monkeys within the temporal lobe, including perirhinal cortex $(\boldsymbol{A})$, superior temporal sulcus $(\boldsymbol{B})$, and bilateral hippocampus $(\boldsymbol{C})$. Other subcortical structures included substantia nigra and thalamus $(\boldsymbol{C}, \boldsymbol{D})$. Clusters were also seen in the parietal and parieto-occipital regions $(\boldsymbol{E}, \boldsymbol{F})$. A representative voxel in dorsal superior temporal sulcus depicts the interaction. The voxel and cluster thresholds were, respectively, $p<0.005$ (uncorrected) and $p<0.05$ (corrected).

Table 4. Group-modified iron accumulation differences with Age

\begin{tabular}{llll}
\hline Region & Voxel T & $\begin{array}{l}\text { Cluster } \\
\text { Size }\end{array}$ & $x, y, z(\mathrm{~mm})$ \\
\hline Left occipital & 4.58 & 11213 & $-17,-19,20$ \\
$\quad$ Left parieto-0ccipital area & 4.08 & & $-23,-4,27$ \\
$\quad$ Left ventral intraparietal area & 3.56 & & $-15,7,26$ \\
Dorsal bank of superior temporal sulcus & 4.46 & 3558 & $26,0,25$ \\
Right hippocampus/dentate gyrus & 3.98 & 13177 & $14,2,14$ \\
$\quad$ Left ventral posterior lateral thalamus & 3.82 & & $-5,7,16$ \\
$\quad$ Right hippocampus & 3.72 & & $-11,0,16$ \\
Right ventral bank of superior temporal sulcus & 3.7 & 1558 & $25,10,6$ \\
$\quad$ Right dorsal bank of superior temporal sulcus & 2.8 & & $20,16,4$ \\
Left perirhinal gyrus & 3.66 & 1353 & $-9,11,-4$ \\
\hline
\end{tabular}

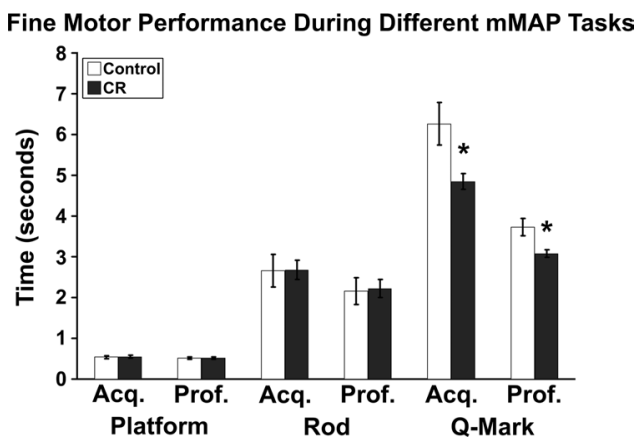

Figure 4. Fine motor performance during mMAP and the effect of Dietary Group. Color bars represent mean time required to complete the fine motor component of the platform, rod, or q-mark task during the acquisition (Acq.) and proficiency (Prof.) phases for control and CR monkeys. Unlike the more simple motor tasks, CR monkeys exhibited faster fine motor performance during both test phases. ${ }^{*} p<0.05$ for ANCOVA at a given phase.
Péran et al., 2007; Xu et al., 2008) and monkeys (Hardy et al., 2005; Cass et al., 2007). Motor deficits also increase with age, and can progress clinically to age-related disorders (Herndon et al., 1997; Lacreuse et al., 1999; Nagahara et al., 2010). In the present study, we confirmed the age-associated increase of iron in a number of brain regions that typically show the most accumulation with age, as indexed by robust negative correlations between Age and T2 in GP and SN (Hallgren and Sourander, 1958; Koeppen, 2003; Haacke et al., 2005).

We also observed a main effect of diet on motor performance and brain iron accumulation in deep brain nuclei. Levels of iron concentration were lower in CR monkeys than controls in GP and SN bilaterally, and left RN. In association with these neural effects, the CR group performed significantly faster than controls on the q-mark task ( 3.08 vs 3.75 s). These task performance speeds are comparable to the values reported by Cass et al. (2007), who used the same motor battery in assessments of middle-aged (15-17 years) and elderly (21-32 years) monkeys. The CR animals in our cohort performed the task at the same level as Cass' middleaged monkeys (3.08 and $\sim 3.25 \mathrm{~s}$, respectively) despite being nearly 10 years older on average. Although these changes in fine motor movement may be influenced by differences in food motivation among CR monkeys (Raman et al., 2007) or attentional processes, these possibilities were controlled for by covarying their performance on both of the simpler platform and rod tasks. Additionally, because we covaried for the effects of age and sex, related variables such as arm strength or sex-specific fine motor differences would not have influenced the results. CR in monkeys and rodents confers protection from age- or substancerelated decline in several motor domains besides fine motor movement, including hand-eye coordination and balance (Duan and Mattson, 1999; Ramsey et al., 2000; Maswood et al., 2004). In humans, comparable deposition in SN pars compacta and changes in motor performance are observed in connection with neurodegenerative diseases, including Parkinson's disease (Gorell et al., 1995) and Alzheimer's disease by itself (Zhu et al., 2009) or with parkinsonism (Brar et al., 2009).

Indeed, our findings complement studies examining several Parkinson's disease-like motor and neural deficits in rodents and rhesus monkeys caused by 1-methyl-4-phenyl1,2,3,6-tetrahydropyridine (MPTP), which induces oxidative and metabolic stress akin to the accumulation of more reactive oxyhydroxy forms of intracellular iron (Tipton and Singer, 1993). Mice or monkeys given MPTP exhibited deficits in motor speed, coordination, and locomotion compared with baseline, but animals on long-term CR recovered more quickly than dietary controls (Duan and Mattson, 1999; Maswood et al., 2004). This improvement corresponded to reduced depletion of dopamine in striatum and more tyrosine hydroxylase-positive neurons in SN and striatum, indicating preservation of dopamine-rich neurons. CR therefore broadly reduces oxidative stress in basal ganglia across species with corresponding benefits in a variety of motor tasks. 
Finally, the influence of age on iron accumulation was positively modulated by $\mathrm{CR}$ in caudate, $\mathrm{SN}$, red nucleus, hippocampus, parietal, and temporal cortices. This iron homeostasis suggests that CR slowed the aging process in these regions. A flattening of the aging slope concurs with previous findings suggesting a protective effect of $\mathrm{CR}$, including gray matter volume preservation in CR monkeys (Colman et al., 2009). Improved motor performance and manual dexterity in the CR animals further underscores the functional significance of these findings. Fine motor deficits in the q-mark task were evident in the split-level correlations with iron accumulation. Increased iron was associated with slower food retrieval time in left SN and left parietal cortex for control but not CR monkeys. However, discerning whether there is high specificity for these neural associations with only motor performance will require further study and is beyond the scope of this report.

Iron homeostasis in the gut and brain is tightly regulated to minimize oxidative damage (Bishop et al., 2007). It is likely that changes in these regulatory systems mediate linear age-related increases in ferritin and perhaps neuromelanin iron sequestration in BG among microglia and, to a much lesser extent, dopaminergic neurons (Zecca et al., 2004a,b). As animals age, the rate of nonenzymatic glycation between proteins and sugars increases, leading to the production of advanced glycation end products (AGEs) and subsequent free radical production (Yan et al., 1996). Mitochondrial dysfunction in electron exchange transport systems also produces free radicals as a function of age (Parker et al., 1994). The generation of reactive oxygen species causes a strong compensatory response of the cell to increase antioxidant activity by upregulating ferritin production to bind excess redox-ready iron and reduce related toxicity (Halliwell and Gutteridge, 1990; Lovell et al., 1995; Young and Woodside, 2001; Grosser et al., 2004; Recalcati et al., 2008; Ansari and Scheff, 2010).

Despite this tightly managed homeostasis, excess iron in the cytosol increasingly becomes integrated with unstable complexes such as hemosiderin and induces oxidative stresswhich may occur due to dysregulated mitochondria-bound transport complexes releasing bound iron (Mastroberardino et al., 2009). Microglia could additionally release proinflammatory cytokines such as interleukin-6 that can act in a paracrine manner on neurons, mediating induction of oxidative stress and diffuse atrophy (Willette et al., 2010). By contrast, there are many more neuromelanin cells in the locus ceruleus, which appear to buffer against excess iron accumulation through chelation (Zecca et al., 2004b). There is also much less iron that accumulates in this area compared with BG, perhaps due to the lack of dopamine-rich neurons that require relatively more iron to drive mitochondrial electron transfer (Mastroberardino et al., 2009).

Although the CR group had slightly higher levels of dietary iron intake, both groups had similar levels of peripheral nonheme iron, suggesting that peripheral uptake did not mediate CR-induced decreases in central iron accumulation. Further, the negative correlation of serum iron and T2 signal in SN points to compensatory changes in central transport or uptake mechanisms. Higher peripheral transferrin induces epithelial cells of the blood-brain barrier to have a reduced capacity both for binding transferrin and for transferring iron ions into the interstitium of brain (Moos, 2002), downregulating central accumulation and potential neurotoxic effects in areas such as SN (Gorell et al., 1995; Zecca et al., 2004a; Unger et al., 2007). CR has been shown to maintain the hypothalamicpituitary-gonadal axis, which in turn maintains perfusion (Wilson et al., 2008). Lifelong 40\% CR in mice improves blood-brain vascular integrity and reduces neuronal loss after induction of thiamine deficiency, which upregulates reactive iron, iron sequestration proteins, and oxidative stress in microglia (Calingasan and Gibson, 2000). CR may likewise reduce age-related blood-brain barrier permeability and parenchymal iron influx.

Other possible mechanisms by which CR could regulate iron accumulation include changes in transferrin receptor binding potentials on neurons (Roskams and Connor, 1990) or alterations in the amount of soluble intracellular iron sequestered into ferritin versus ion use in protein synthesis or efflux into interstitial CSF (Bradbury, 1997). Finally, CR may also act intracellularly by lowering energy metabolism (Masoro et al., 1992; Fontana, 2009), subsequently downregulating AGEs, oxidative stress (Zainal et al., 2000; Kayo et al., 2001), and possibly a compensatory increase in L-ferritin binding. It was not feasible to directly examine histological tissue to test these hypotheses in this ongoing, longitudinal study. Such samples will be collected at time of death to examine ferritin binding potential and other intracellular mechanisms.

Monkeys on a CR diet show reduced iron accumulation in the GP, SN, and temporal cortex compared with controls, in addition to showing less correlation between iron concentration and Age in GP and SN. Consumption of a CR diet from middle age slows the rate of iron accumulation in parietal and temporal cortices, GP, SN, and RN. CR monkeys showed preservation of motor performance, appearing similar to published reports of monkeys 10 years younger, which were associated with the iron determination in brain areas associated with motor function. Our observations suggest that the CR benefit of reduced iron deposition and preserved motor function may indicate neural protection similar to effects described previously in aging rodent and primate species. 


\section{References}

Anderson RM, Shanmuganayagam D, Weindruch R (2009) Caloric restriction and aging: studies in mice and monkeys. Toxicol Pathol 37:47-51.

Ansari MA, Scheff SW (2010) Oxidative stress in the progression of Alzheimer disease in the frontal cortex. J Neuropathol Exp Neurol 69:155-167.

Anson RM, Guo Z, de Cabo R, Iyun T, Rios M, Hagepanos A, Ingram DK, Lane MA, Mattson MP (2003) Intermittent fasting dissociates beneficial effects of dietary restriction on glucose metabolism and neuronal resistance to injury from calorie intake. Proc Natl Acad Sci U S A 100:6216-6220.

Ashburner J, Friston KJ (2000) Voxel-based morphometry-the methods. Neuroimage 11:805-821.

Bartzokis G, Beckson M, Hance DB, Marx P, Foster JA, Marder SR (1997) MR evaluation of age-related increase of brain iron in young adult and older normal males. Magn Reson Imaging 15:29-35.

Bartzokis G, Tishler TA, Lu PH, Villablanca P, Altshuler LL, Carter M, Huang D, Edwards N, Mintz J (2007) Brain ferritin iron may influence age- and gender-related risks of neurodegeneration. Neurobiol Aging 28:414-423.

Ben-Shachar D, Yehuda S, Finberg JP, Spanier I, Youdim MB (1988) Selective alteration in blood-brain barrier and insulin transport in irondeficient rats. J Neurochem 50:1434-1437.

Bishop GM, Smith MA, LaManna JC, Wilson AC, Perry G, Atwood CS (2007) Iron homeostasis is maintained in the brain, but not the liver, following mild hypoxia. Redox Rep 12:257-266.

Bizzi A, Brooks RA, Brunetti A, Hill JM, Alger JR, Miletich RS, Francavilla TL, Di Chiro G (1990) Role of iron and ferritin in MR imaging of the brain: A study in primates at different field strengths. Radiology 177:59-65.

Bradbury MW (1997) Transport of iron in the blood-brain-cerebrospinal fluid system. J Neurochem 69:443-454.

Brar S, Henderson D, Schenck J, Zimmerman EA (2009) Iron accumulation in the substantia nigra of patients with Alzheimer disease and parkinsonism. Arch Neurol 66:371-374.

Calingasan NY, Gibson GE (2000) Dietary restriction attenuates the neuronal loss, induction of heme oxygenase-1 and blood-brain barrier breakdown induced by impaired oxidative metabolism. Brain Res 885:62-69.

Cass WA, Grondin R, Andersen AH, Zhang Z, Hardy PA, Hussey-Andersen LK, Rayens WS, Gerhardt GA, Gash DM (2007) Iron accumulation in the striatum predicts aging-related decline in motor function in rhesus monkeys. Neurobiol Aging 28:258-271.

Cattell RB (1966) The scree test for the number of factors. Multivariate Behav Res 1:245-276.

Colman RJ, Beasley TM, Allison DB, Weindruch R (2008) Attenuation of sarcopenia by dietary restriction in rhesus monkeys. J Gerontol A Biol Sci Med Sci 63:556-559.

Colman RJ, Anderson RM, Johnson SC, Kastman EK, Kosmatka KJ, Beasley TM, Allison DB, Cruzen C, Simmons HA, Kemnitz JW, Weindruch R (2009) Caloric restriction delays disease onset and mortality in rhesus monkeys. Science 325:201-204.

Dhenain M, Michot JL, Volk A, Picq JL, Boller F (1997) T2-weighted MRI studies of mouse lemurs: a primate model of brain aging. Neurobiol Aging 18:517-521.

Drayer B, Burger P, Darwin R, Riederer S, Herfkens R, Johnson G (1986) MRI of brain iron. Am J Roentgenol 147:103-110.

Duan W, Mattson MP (1999) Dietary restriction and 2-deoxyglucose administration improve behavioral outcome and reduce degeneration of dopaminergic neurons in models of Parkinson's disease. J Neurosci Res $57: 195-206$

Fontana L (2009) The scientific basis of caloric restriction leading to longer life. Curr Opin Gastroenterol 25:144-150.

Forman SD, Cohen JD, Fitzgerald M, Eddy WF, Mintun MA, Noll DC (1995) Improved assessment of significant activation in functional magnetic resonance imaging (fMRI): use of a cluster-size threshold. Magn Reson Med 33:636-647.

Forster MJ, Sohal BH, Sohal RS (2000) Reversible effects of long-term caloric restriction on protein oxidative damage. J Gerontol A Biol Sci Med Sci 55:B522-B5229.

Gash DM, Zhang Z, Umberger G, Mahood K, Smith M, Smith C, Gerhardt GA (1999) An automated movement assessment panel for upper limb motor functions in rhesus monkeys and humans. J Neurosci Methods 89:111-117.

Gelman N, Gorell JM, Barker PB, Savage RM, Spickler EM, Windham JP, Knight RA (1999) MR imaging of human brain at 3.0 T: Preliminary report on transverse relaxation rates and relation to estimated iron content. Radiology 210:759-767.

Gorell JM, Ordidge RJ, Brown GG, Deniau JC, Buderer NM, Helpern JA (1995) Increased iron-related MRI contrast in the substantia nigra in Parkinson's disease. Neurology 45:1138-1143.

Gresl TA, Colman RJ, Roecker EB, Havighurst TC, Huang Z, Allison DB, Bergman RN, Kemnitz JW (2001) Dietary restriction and glucose regulation in aging rhesus monkeys: a follow-up report at $8.5 \mathrm{yr}$. Am J Physiol Endocrinol Metab 281:E757-E765.

Gresl TA, Colman RJ, Havighurst TC, Byerley LO, Allison DB, Schoeller DA, Kemnitz JW (2003) Insulin sensitivity and glucose effectiveness from three minimal models: effects of energy restriction and body fat in adult male rhesus monkeys. Am J Physiol Regul Integr Comp Physiol 285:R1340-R1354.

Grosser N, Oberle S, Berndt G, Erdmann K, Hemmerle A, Schröder H (2004) Antioxidant action of L-alanine: heme oxygenase- 1 and ferritin as possible mediators. Biochem Biophys Res Commun 314:351-355.

Haacke EM, Cheng NYC, House MJ, Liu Q, Neelavalli J, Ogg RJ, Khan A, Ayaz M, Kirsch W, Obenaus A (2005) Imaging iron stores in the brain using magnetic resonance imaging. Magn Reson Imaging 23:1-25.

Hallgren B, Sourander P (1958) The effect of age on the non-haemin iron in the human brain. J Neurochem 3:41-51.

Halliwell B, Gutteridge JM (1990) Role of free radicals and catalytic metal ions in human disease: an overview. Methods Enzymol 186:1-85.

Hardy PA, Gash D, Yokel R, Andersen A, Ai Y, Zhang Z (2005) Correlation of R2 with total iron concentration in the brains of rhesus monkeys. J Magn Reson Imaging 21:118-127.

Hayasaka S, Phan KL, Liberzon I, Worsley KJ, Nichols TE (2004) Nonstationary cluster-size inference with random field and permutation methods. Neuroimage 22:676-687.

Herndon JG, Moss MB, Rosene DL, Killiany RJ (1997) Patterns of cognitive decline in aged rhesus monkeys. Behav Brain Res 87:25-34

House MJ, St Pierre TG, Kowdley KV, Montine T, Connor J, Beard J, Berger J, Siddaiah N, Shankland E, Jin LW (2007) Correlation of proton transverse relaxation rates (R2) with iron concentrations in postmortem brain tissue from Alzheimer's disease patients. Magn Reson Med 57:172-180.

Kayo T, Allison DB, Weindruch R, Prolla TA (2001) Influences of aging and caloric restriction on the transcriptional profile of skeletal muscle from rhesus monkeys. Proc Natl Acad Sci U S A 98:5093-5098.

Kemnitz JW, Weindruch R, Roecker EB, Crawford K, Kaufman PL, Ershler WB (1993) Dietary restriction of adult male rhesus monkeys: design, methodology, and preliminary findings from the first year of study. J Gerontol 48:B17-B26.

Koeppen AH (2003) A brief history of brain iron research. J Neurol Sci 207:95-97.

Lacreuse A, Herndon JG, Killiany RJ, Rosene DL, Moss MB (1999) Spatial cognition in rhesus monkeys: Male superiority declines with age. Hormones Behav 36:70-76.

Lovell MA, Ehmann WD, Butler SM, Markesbery WR (1995) Elevated thiobarbituric acid-reactive substances and antioxidant enzyme activity in the brain in Alzheimer's disease. Neurology 45:1594-1601.

Martin WR, Ye FQ, Allen PS (1998) Increasing striatal iron content associated with normal aging. Mov Disord 13:281-286.

Masoro EJ (2005) Overview of caloric restriction and ageing. Mech Ageing Dev 126:913-922.

Masoro EJ, McCarter RJ, Katz MS, McMahan CA (1992) Dietary restriction alters characteristics of glucose fuel use. J Gerontol 47:B202-B208.

Maswood N, Young J, Tilmont E, Zhang Z, Gash DM, Gerhardt GA, Grondin R, Roth GS, Mattison J, Lane MA, Carson RE, Cohen RM, Mouton PR, Quigley C, Mattson MP, Ingram DK (2004) Caloric restriction increases neurotrophic factor levels and attenuates neurochemical and behavioral deficits in a primate model of Parkinson's disease. Proc Natl Acad Sci U S A 101:18171-18176.

Mattison JA, Roth GS, Lane MA, Ingram DK (2007) Dietary restriction in aging nonhuman primates. Interdiscip Top Gerontol 35:137-158.

McLaren DG, Kosmatka KJ, Oakes TR, Kroenke CD, Kohama SG, Matochik JA, Ingram DK, Johnson SC (2009) A population-average MRI-based atlas collection of the rhesus macaque. Neuroimage 45:52-59.

McLaren DG, Kosmatka KJ, Kastman EK, Bendlin BB, Johnson SC (2010) Rhesus macaque brain morphometry: a methodological comparison of voxel-wise approaches. Methods 50:157-165.

Mechelli A, Price KJ, Friston KJ, Ashburner J (2005) Voxel-based mor- 
phometry of the human brain: methods and applications. Curr Med Imaging Rev 1:1-9.

Moos T (2002) Brain iron homeostasis. Dan Med Bull 49:279-301.

Mouton PR, Chachich ME, Quigley C, Spangler E, Ingram DK (2009) Caloric restriction attenuates amyloid deposition in middle-aged dtg APP/ PS1 mice. Neurosci Lett 464:184-187.

Nagahara AH, Bernot T, Tuszynski MH (2010) Age-related cognitive deficits in rhesus monkeys mirror human deficits on an automated test battery. Neurobiol Aging 31:1020-1031.

Parker WD Jr, Parks J, Filley CM, Kleinschmidt-DeMasters BK (1994) Electron transport chain defects in Alzheimer's disease brain. Neurology 44:1090-1096.

Péran P, Hagberg G, Luccichenti G, Cherubini A, Brainovich V, Celsis P, Caltagirone C, Sabatini U (2007) Voxel-based analysis of R2* maps in the healthy human brain. J Magn Reson Imaging 26:1413-1420.

Poon CS, Henkelman RM (1992) Practical T2 quantitation for clinical applications. J Magn Reson Imaging 2:541-553.

Qin W, Yang T, Ho L, Zhao Z, Wang J, Chen L, Zhao W, Thiyagarajan M, MacGrogan D, Rodgers JT, Puigserver P, Sadoshima J, Deng H, Pedrini S, Gandy S, Sauve AA, Pasinetti GM (2006) Neuronal SIRT1 activation as a novel mechanism underlying the prevention of Alzheimer disease amyloid neuropathology by calorie restriction. J Biol Chem 281:21745-21754.

Raman A, Ramsey JJ, Kemnitz JW, Baum ST, Newton W, Colman RJ, Weindruch R, Beasley MT, Schoeller DA (2007) Influences of calorie restriction and age on energy expenditure in the rhesus monkey. Am J Physiol Endocrinol Metab 292:E101-E106.

Recalcati S, Invernizzi P, Arosio P, Cairo G (2008) New functions for an iron storage protein: the role of ferritin in immunity and autoimmunity. J Autoimmun 30:84-89.

Ribeiro LC, Quincozes-Santos A, Leite MC, Abib RT, Kleinkauf-Rocha J, Biasibetti R, Rotta LN, Wofchuk ST, Perry ML, Gonçalves CA, Gottfried C (2009) Caloric restriction increases hippocampal glutamate uptake and glutamine synthetase activity in Wistar rats. Neurosci Res 64:330-334.

Richardson DR, Ponka P (1997) The molecular mechanisms of the metabolism and transport of iron in normal and neoplastic cells. Biochim Biophys Acta 1331:1-40.

Ridgway GR, Henley SM, Rohrer JD, Scahill RI, Warren JD, Fox NC (2008) Ten simple rules for reporting voxel-based morphometry studies. Neuroimage 40:1429-1435.

Roskams AJ, Connor JR (1990) Aluminum access to the brain: a role for transferrin and its receptor. Proc Natl Acad Sci U S A 87:9024-9027.

Saleem KS, Logothetis NK (2007) A combined MRI and histology atlas of the rhesus monkey brain in stereotaxic coordinates. London: Academic.

Schenck JF, Zimmerman EA (2004) High-field magnetic resonance imaging of brain iron: birth of a biomarker? NMR Biomed 17:433-445.

Stranahan AM, Lee K, Martin B, Maudsley S, Golden E, Cutler RG, Mattson MP (2009) Voluntary exercise and caloric restriction enhance hippocampal dendritic spine density and BDNF levels in diabetic mice. Hippocampus 19:951-961.

Tipton KF, Singer TP (1993) Advances in our understanding of the mechanisms of the neurotoxicity of MPTP and related compounds. J Neurochem 61:1191-1206.
Ueda F, Raja KB, Simpson RJ, Trowbridge IS, Bradbury MW (1993) Rate of $59 \mathrm{Fe}$ uptake into brain and cerebrospinal fluid and the influence thereon of antibodies against the transferrin receptor. J Neurochem 60:106-113.

Unger EL, Beard JL, Jones BC (2007) Iron regulation in C57BLI6 and DBA/2 mice subjected to iron overload. Nutr Neurosci 10:89-95.

Wanagat J, Allison DB, Weindruch R (1999) Caloric intake and aging: mechanisms in rodents and a study in nonhuman primates. Toxicol Sci 52:35-40.

Weindruch R, Walford RL (1988) The retardation of aging and disease by dietary restriction. Springfield, IL: Charles C Thomas.

Willette AA, Bendlin BB, McLaren DG, Canu E, Kastman EK, Kosmatka KJ, Xu G, Field AS, Alexander AL, Colman RJ, Weindruch RH, Coe CL, Johnson SC (2010) Age-related changes in neural volume and microstructure associated with interleukin- 6 are ameliorated by a calorierestricted diet in old rhesus monkeys. Neuroimage 51:987-994.

Wilson AC, Clemente L, Liu T, Bowen RL, Meethal SV, Atwood CS (2008) Reproductive hormones regulate the selective permeability of the bloodbrain barrier. Biochim Biophys Acta 1782:401-407.

$\mathrm{Xu}$ J, Knutson MD, Carter CS, Leeuwenburgh C (2008) Iron accumulation with age, oxidative stress and functional decline. PLoS One 3:e2865.

Xu X, Wang Q, Zhang M (2008) Age, gender, and hemispheric differences in iron deposition in the human brain: an in vivo MRI study. Neuroimage 40:35-42.

Yan SD, Chen X, Fu J, Chen M, Zhu H, Roher A, Slattery T, Zhao L, Nagashima M, Morser J, Migheli A, Nawroth P, Stern D, Schmidt AM (1996) RAGE and amyloid-beta peptide neurotoxicity in Alzheimer's disease. Nature 382:685-691.

Young IS, Woodside JV (2001) Antioxidants in health and disease. J Clin Pathol 54:176-186.

Zainal TA, Oberley TD, Allison DB, Szweda LI, Weindruch R (2000) Caloric restriction of rhesus monkeys lowers oxidative damage in skeletal muscle. FASEB J 14:1825-1836.

Zecca L, Gallorini M, Schünemann V, Trautwein AX, Gerlach M, Riederer P, Vezzoni P, Tampellini D (2001) Iron, neuromelanin and ferritin content in the substantia nigra of normal subjects at different ages: consequences for iron storage and neurodegenerative processes. J Neurochem 76:1766-1773.

Zecca L, Youdim MB, Riederer P, Connor JR, Crichton RR (2004a) Iron, brain ageing and neurodegenerative disorders. Nat Rev Neurosci 5:863-873.

Zecca L, Stroppolo A, Gatti A, Tampellini D, Toscani M, Gallorini M, Giaveri G, Arosio P, Santambrogio P, Fariello RG, Karatekin E, Kleinman MH, Turro N, Hornykiewicz O, Zucca FA (2004b) The role of iron and copper molecules in the neuronal vulnerability of locus coeruleus and substantia nigra during aging. Proc Natl Acad Sci U S A 101:9843-9848.

Zhang Y, Brady M, Smith S (2001) Segmentation of brain MR images through a hidden Markov random field model and the expectationmaximization algorithm. IEEE Trans Med Imaging 20:45-57.

Zhu WZ, Zhong WD, Wang W, Zhan CJ, Wang CY, Qi JP, Wang JZ, Lei T (2009) Quantitative MR phase-corrected imaging to investigate increased brain iron deposition of patients with Alzheimer disease. Radiology 253:497-504.

\section{Correction}

\section{Correction: Kastman et al., A Calorie-Restricted Diet Decreases Brain Iron Accumulation and Preserves Motor Performance in Old Rhesus Monkeys}

In the original version of the article "A Calorie-Restricted Diet Decreases Brain Iron Accumulation and Preserves Motor Performance in Old Rhesus Monkeys" by Erik K. Kastman, Auriel A. Willette, Christopher L. Coe, Barbara B. Bendlin, Kris J. Kosmatka, Donald G. McLaren, Guofan Xu, Elisa Canu, Aaron S. Field, Andrew L. Alexander, Mary Lou Voytoko, T. Mark Beasley, Ricki J. Colman, Richard H. Weindruch, and Sterling C. Johnson, which appeared on pages 7940-7947 of the June 9, 2010 issue, the term "T2" was incorrectly replaced with "R2" in many places. This was a semantic error only. All the data and the analyses were based on T2-relaxation maps and all of the interpretations and conclusions in the article hold once this error is corrected. Because of the potential for misinterpretation and the number of places where the term was misused, this corrected version of the article is being published.

DOI: 10.1523/JNEUROSCI.2553-12.2012 